\title{
Primeramente Dios, yo la alivio. El legado de Lorenzo Amaya en Nueva York
}

\author{
Carmen Molina Tamacas \\ Antropóloga y periodista radicada en Estados Unidos \\ cmolinatamacas@gmail.com
}

\section{Resumen}

En 2011, la Editorial Campana de Nueva York publicó una recopilación de testimonios de parteras salvadoreñas - Primeramente Dios, yo la aliviorealizada por el investigador Lorenzo Amaya Guevara (1942-2015).

El libro fue realizado en coautoría con la lingüista española Paquita SuárezCoalla y es parte del trabajo realizado a finales de los años 80 y principios de los 90 en Ciudad Barrios, San Miguel, por el grupo Visión Cuscatleca. Las historias de vida incluyen leyendas, dietas, rituales, oraciones, cosmovisión y medicina natural.

Palabras clave: parteras, antropología, etnografía, medicina natural, parto, San Miguel.

\begin{abstract}
In 2011, New York Editorial Campana published a compilation of testimonies of Salvadoran midwives, conducted by researcher Lorenzo Amaya Guevara (1942-2015).

The book was made co-authored with the Spanish linguist Paquita SuárezCoalla and is part of the work done in the late 80s and early 90s in Ciudad Barrios, San Miguel, by Vision Cuscatleca Organisation. Life stories include legends, diet, rituals, prayers, worldview and natural medicine.
\end{abstract}

Key words: midwives, Anthropology, Ethnography, natural medicine, birth, San Miguel. 


\section{Introducción}

Soy originaria de este pueblo de Ciudad Barrios. Nací cerca del cantón Llano del Ángel. Hoy, que ya tengo 93 años, vivo todavía con mi esposo en esta casita y cerca de aquí viven mis hijas y nietos (...). En mi casa vivía un gran familión. Sí, había unas cuantas muchachas. Me acuerdo que vivíamos allá por donde está un cerro que le dicen el Bonete. Ese cerro es encantado. Allí vivía un duende.

Así inicia el relato de María Padilla, quien nació en un remoto cantón de Ciudad Barrios, San Miguel, en 1902; y dos años antes de morir, en 1992, compartió el relato de su vida como partera.

La suya es una de 24 historias recopiladas por Lorenzo Amaya Guevara, reconocido por su gran trayectoria como investigador de la cultura salvadoreña, en particular de la producción del añil.

Amaya murió el 24 de julio de 2015, dejando un legado como parte de su trabajo de casi cuatro décadas en la investigación cultural gubernamental. Tras un repentino derrame cerebral, permaneció varios meses delicado de salud hasta que sus fuerzas se apagaron.

Admiré la humildad de Lorenzo Amaya y su capacidad para compartir conocimiento, cuando en 2005 fue mi catedrático de Tierra, Cultura y Medioambiente en la Escuela de Antropología de la Universidad Tecnológica (Utec), aunque antes ya formaba parte de la lista de fuentes de información para el equipo de la ahora extinta Revista Dominical de La Prensa Gráfica.

\section{El origen de Primeramente Dios...}

En marzo de este año, el Consulado de El Salvador en Manhattan organizó un conversatorio por el aniversario del martirio de monseñor Óscar Arnulfo Romero. Ese día llegaron a mis manos dos ejemplares de Primeramente Dios, yo la alivio, un libro publicado en Nueva York por la Editorial Campana, cuyo autor es Lorenzo Amaya.

Había esperado con ansias ese momento, prometido meses atrás por Aracely Sánchez, una salvadoreña que vive en Brooklyn. En un conversatorio, organizado también por el Consulado de salvadoreños en el exterior, me contó que había ayudado a hacer realidad el sueño de Lorenzo, para que la recopilación de entrevistas de parteras migueleñas viera la luz. Llamó mucho la atención por el tema, pero especialmente porque aún cuatro años después el libro es desconocido en El Salvador.

A finales de los años 80, Aracely y Lorenzo fundaron la organización Visión Cuscatleca, que tiene como objetivo investigar y promover las expresiones artísticas y el folclore salvadoreño. 


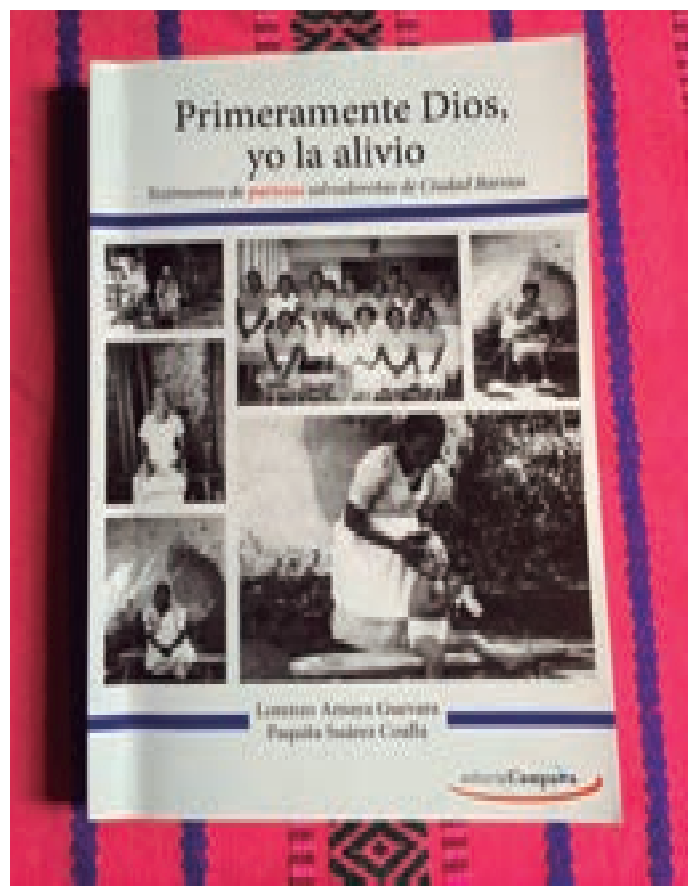

Aracely, en una conversación posterior a la muerte de él, recordó que

Lorenzo y yo trabajamos desde los años ochenta en diferentes proyectos de investigación cultural, y el libro fue uno de esos proyectos que con mucho esfuerzo y dedicación logramos publicar. Dentro del abanico de proyectos, antes de que yo saliera de El Salvador, ya teníamos en proceso el trabajo sobre parteras.

En la nota introductoria del libro, Amaya explicó que Visión Cuscatleca estuvo formada por estudiantes de diversas disciplinas y con intereses afines: música, filosofía, psicología social, sociología. "Nuestro objetivo era rescatar, promover y difundir nuestros valores ancestrales."

A finales de los años 80 — precisó- propuso al grupo

hacer una recopilación de testimonios de parteras de la zona oriental del país, especificamente de Ciudad Barrios, por ser la zona menos estudiada, y por ser la partera la portadora de un amplio bagaje cultural (...). El análisis de las condiciones de vida de la mujer en el área oriental nos hizo crear también el trabajo musical "Mujer campesina" para conmemorar el vigésimo aniversario del Año Internacional de la Mujer en 1995. 
Aún después de haber emigrado, Aracely mantuvo contacto casi permanente con Amaya y el ritmo de trabajo. Ella dice:

Participé en la recolección de las historias, leyendo una a una, financiando los costos de operación necesarios para actualizar la información de los testimonios, hasta ser el puente de comunicación entre la editora, la editorial y Lorenzo cuando era necesario.

Algunas de las entrevistas fueron realizadas en el centro de salud de Ciudad Barrios y otras en los cantones de ese municipio. La metodología usada -indicó Amaya - fue simple: mostrar mucho interés por el trabajo de la partera y lograr un alto grado de confianza, para que esta pudiera hablar sin recelos de su historia de vida, de su experiencia en la atención de partos y de los tratamientos con plantas y fórmulas mágico-religiosas que utilizaban para curar algunas enfermedades en el parto y el posparto, así como para el tratamiento de etno-enfermedades como el ojo, el susto, el pujo, el empacho y la mollera.

Dentro de todo ese conocimiento ancestral, Amaya recolectó nombres de plantas usadas durante el embarazo, el alumbramiento y el posparto; oraciones, rituales, aplicación del conocimiento astronómico, particularmente el de las fases de la Luna. Entre las plantas más mencionadas por las parteras figuran las semillas de San Pedro, raíces de culantro y el limón, la canela, el culantrillo de hacer escobas y la hierba de Rosario, entre muchas otras. Sobre las diversas tradiciones orales de la región destaca la de un duende, ya que aseguran que vive en un cerro y cortejaba a las niñas.

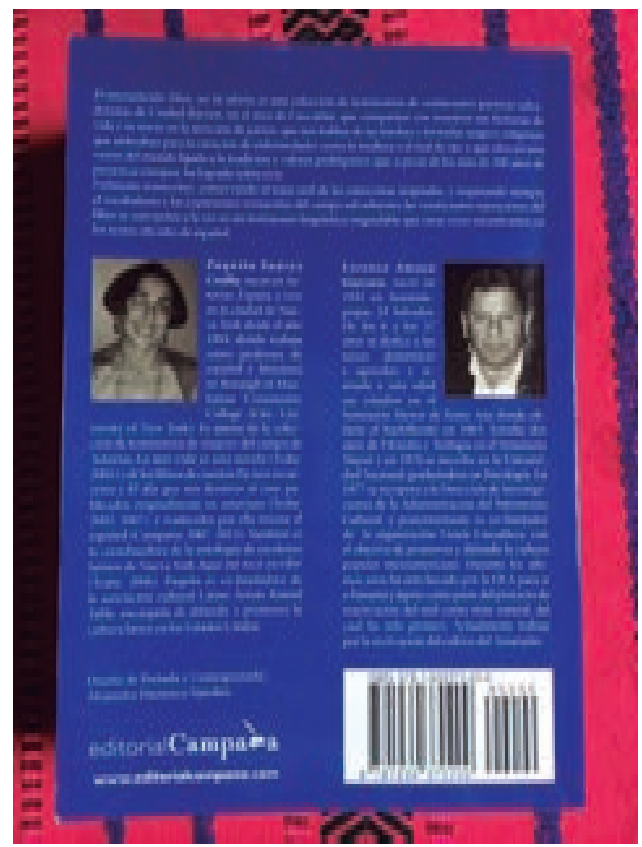

La principal virtud del autor es devolver el calor humano y respeto con el cual fue recibido por estas mujeres, algunas de edad avanzada y sin escolaridad, pero autodidactas.

Como investigador comprometido, el autor también hace una denuncia: pese al trabajo que realizan "las que de lo que no tienen aún le dan a las parturientas más pobres", las matronas no cuentan con un salario mínimo que contrarreste su escasez.

Los testimonios de estas mujeres reflejan con toda objetividad el sufrimiento, la pobreza y el abandono en el que vive el campesino de nuestro país en las áreas de salud, educación y trabajo. 


\section{El legado en un libro}

El libro Primeramente Dios, yo la alivio recoge los testimonios de María Padía Padilla, 93 años; Francisca Carranza Posada, 89; Cayetana Carranza, 84; María Aguilar Pineda, 79; Mirtala Recinos, 78; Lucila López, 75; Guadalupe Centeno Carranza, 74; Carmen Alvarado de Avelar, 70; Fidelina Rodríguez Márquez, 68; Sebastiana Medardo Sorto y Albertina Escamilla, 67; Fidencia Hernández Ortiz, 64; Martina Guevara de Chicas, 63; Carmen Romero, 60; Bernardina Ayala; Rosa Hernández, 59; Aminta Campos, 56; María Hernández Ortiz, 52; María Dionisia Martínez y Bibiana Iglesias, 50; Rosa Chicas y Blanca Nieves Medrano, 49; Ana Julia Posada Lovo, 46 e Isabel Lovo Carranza, 36.

Cada historia - que no excede las diez páginas cada una - incluye un retrato. Por ejemplo, la señora Padía aparece sentada en una silla con las manos sobre las rodillas, frente a la entrada de su humilde vivienda de bahareque. Otras, como Mirtala Recinos y María Aguilar, fueron retratadas con niños en brazos. Los relatos están llenos de detalles que deben ser el punto de partida para estudios antropológicos sobre la sabiduría ancestral y las prácticas sanitarias de las zonas rurales.

Respecto al trabajo de campo, Aracely Sánchez dijo: "Recuerdo a Lorenzo muy dedicado, respetuoso de las historias de cada una de las parteras, entusiasmado con el trabajo y tomando muchos de sus fines de semana y días feriados para viajar hasta Ciudad Barrios".

La obra se publicó en Nueva York con la ayuda de varias personas, entre ellas Paquita Suárez-Coalla, catedrática de español y literatura en la Universidad de la Ciudad de Nueva York (CUNY). Con el título "Nos queda la historia", ella hace una presentación del libro, enfatizando la satisfacción por haber participado en la edición de los testimonios recopilados por Lorenzo Amaya. Dice Paquita:

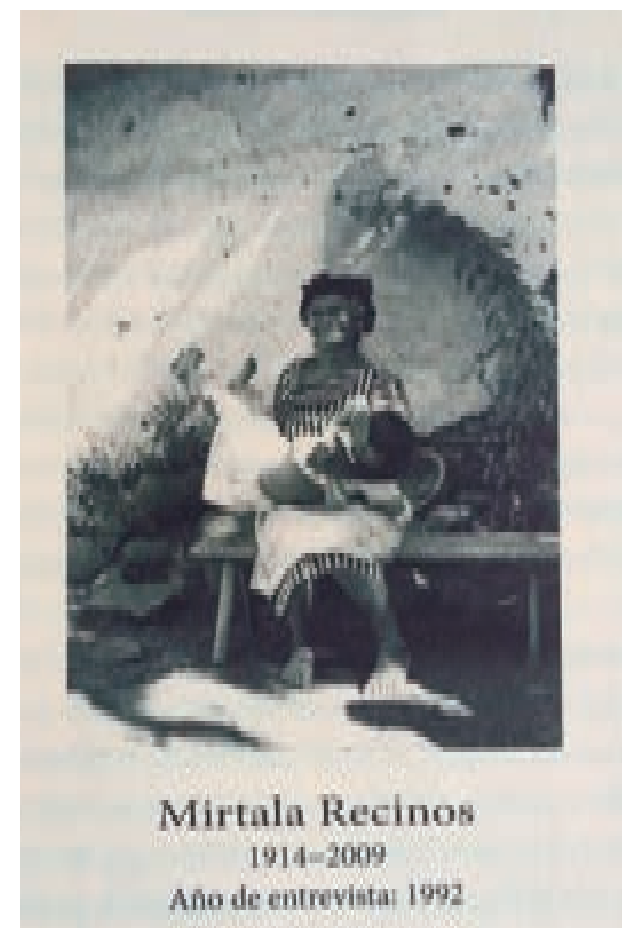

Supe de él, y de su trabajo, por nuestra amiga común Aracely Sánchez, a quien conocí en el 2004 en Nueva York. Enseguida me interesé por lo que estaba haciendo, porque coincidía con un trabajo de recopilación de testimonios que yo misma había hecho años atrás con mujeres campesinas 
del norte de España, de donde soy originalmente, y porque me parece de enorme importancia recoger las voces de aquellos, y sobre todo de aquellas, que cualquier sociedad, sin importar el pais, siempre ignoran y arrinconan, indicó Suárez-Coalla vía comunicación electrónica.

La edición fue un proceso "absolutamente satisfactorio, desde el punto de vista antropológico y, en mi caso, lingüístico", afirma Paquita y relata lo siguiente:

Desde el principio me quedé fascinada no solo con las historias, sino con la lengua - tal vez haya influido en gran medida el hecho de ser escritora $y$ lingüista -; una lengua que no conocía, con un vocabulario de enorme riqueza que no aparecía en los diccionarios oficiales y que tuve que ir consultando pacientemente con Lorenzo. Al principio habia decidido incluir un glosario en el que aparecieran todas aquellas voces que, a partir de mi propio desconocimiento, podrían resultar desconocidas para el lector, pero al final -y me alegro de ello-decidi no hacerlo por la simple razón de que cuando se trata de la lengua que hablan las clases con poder nadie la traduce; y su manera de hablar, que se considera la norma, el canon lingüistico que se debe seguir, sirve para marcar distancia entre el que conoce ese lenguaje y el que no, y el que no lo conoce se considera ignorante, pero nunca se habla de la ignorancia enorme del que no sabe términos populares, o desdeña las formas de hablar del pueblo. En este caso, $y$ al tener en cuenta que la mayoría de las parteras son indigenas, como lo son sus expresiones, ligadas a lenguas prehispánicas que felizmente se han conservado en su boca, el desconocimiento es además una postura de clase que resulta hiriente. Por eso, por respeto, fue que decidí no 'traducir' su lengua, porque tan canónica es esa manera de expresarse como la de las clases pudientes más vinculadas a la herencia de la conquista española. Me gustaría, de todos modos, que, por respeto, la Real Academia de la Lengua Española en El Salvador fuera lo suficientemente inteligente como para no escamotearles el lugar que se merecen en las preciosas páginas de su diccionario. El libro de testimonios es por tanto, además de todo, una joya lingüística que espero se tome como referencia para rescatar la auténtica lengua salvadoreña.

Una vez acababa un trabajo de edición, Paquita lo enviaba a Lorenzo para que le diera su parecer al respecto. "Recuerdo sobre todo que siempre fue una persona muy agradecida con mi colaboración. Aunque me hubiera gustado, nunca llegué a conocerlo, pero nuestro trabajo a través de internet fue cordial y lleno de compenetración".

No es extraño que en Nueva York se encuentren libros publicados en y sobre El Salvador, pero que, paradójicamente, no puedan encontrarse allá. La Biblioteca Pública conserva en sus anaqueles verdaderas joyas, muchas de ellas antiguas, por lo que se requiere permiso especial para lograr su acceso. ¿Por qué se 
publicó Primeramente Dios, yo la alivio en Nueva York? Paquita ha publicado dos títulos con la Editorial Campana: El día que nos llevaron al cine y otros cuentos y la edición en inglés de Para que no se me olvide. En relación con el interrogante, la autora puntualizó:

Yo he estado en contacto con Campana desde su creación y estaba segura de que este trabajo encajaba perfectamente en la línea de la editorial. Cuando hablé con su editor, Mario Picayo, aceptó con gusto la propuesta e hizo todo lo posible para que el libro se publicara. Yo nunca he estado en El Salvador, no tengo ningún contacto allá y no hubiera podido hacer mucho para que este trabajo viera la luz en el país al que se debe. Es cierto que tendría que haberse publicado allá, o haber recibido un mayor apoyo en El Salvador que el que ha recibido. Es una lástima, una gran lástima, que Lorenzo se haya muerto sin haber visto el libro en su país. Que se publique ahora, si es que se publica, es una especie de homenaje a su labor, pero qué mal que la gente tenga que morirse para que se acuerden de lo que hizo. Con esto no quiero decir que solo pase en El Salvador. Ojalá fuera así porque entonces el problema sería pequeño; lamentablemente pasa en todas partes.

La primera edición del libro - firmada por Lorenzo Amaya y Paquita SuárezCoalla - fue publicada en octubre de 2011.

Lorenzo recibió un lote de los libros en El Salvador de manos de la poeta salvadoreña radicada en Nueva York, Juana Ramos, y la poeta argentina Margarita Drago, quienes viajaron para participar en el Primer Festival de Poesía de Occidente, en Chalchuapa, Santa Ana.

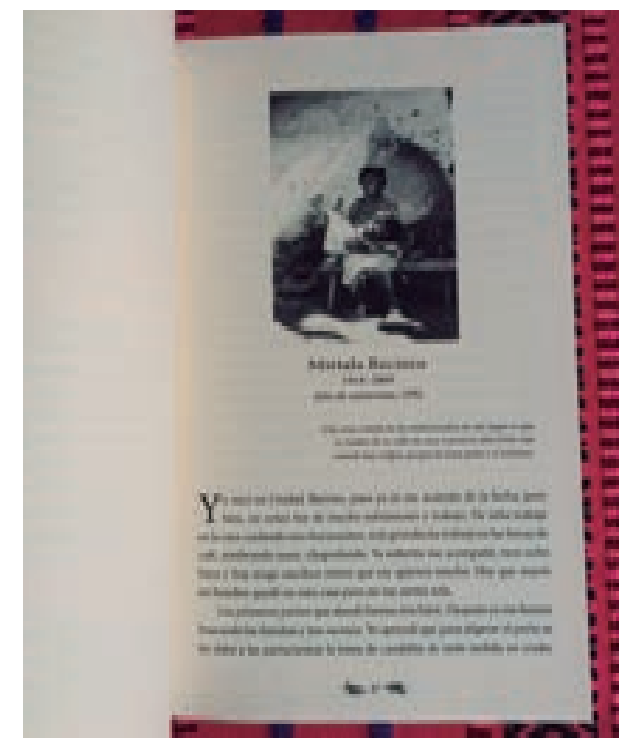




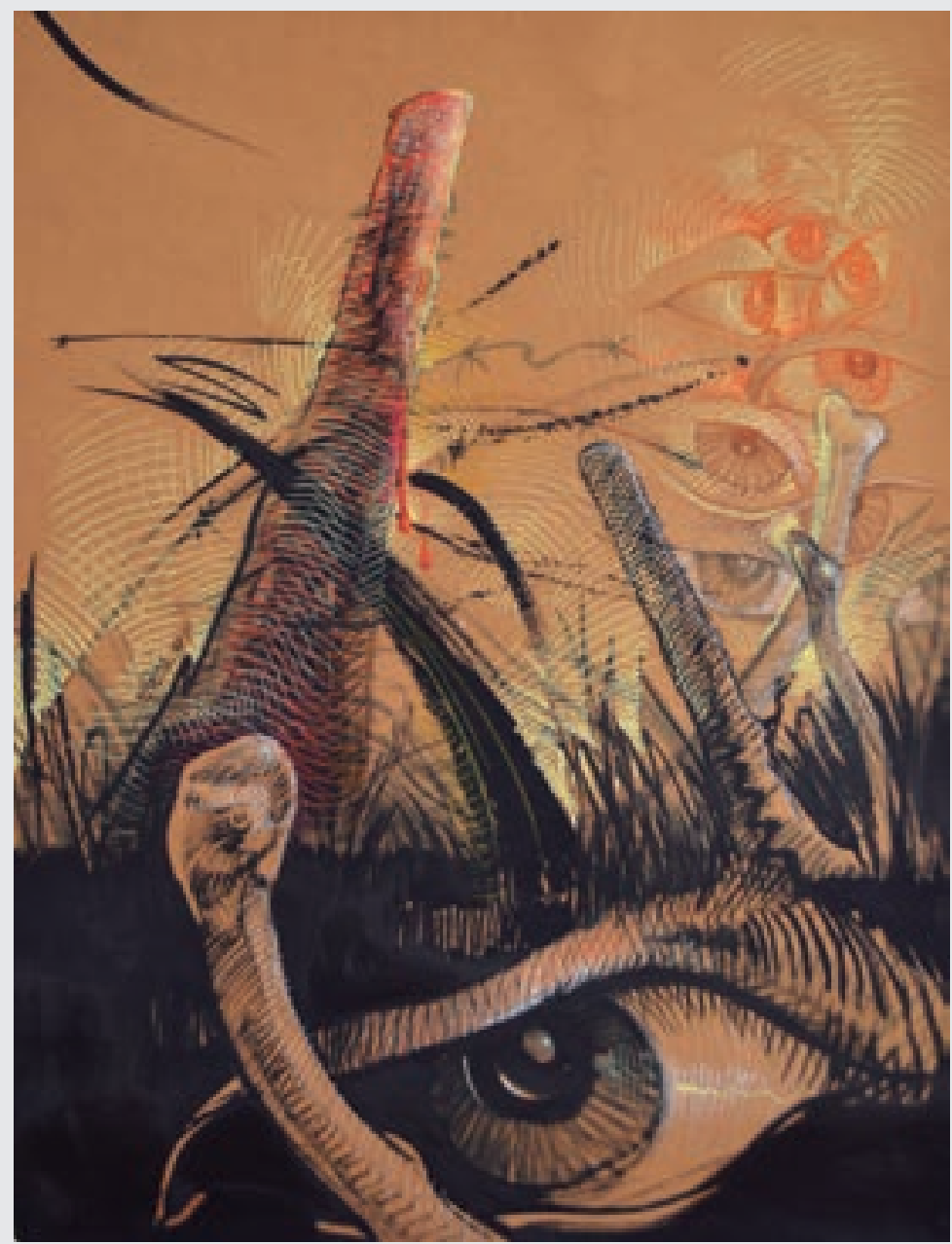

